\title{
Kerr Black Holes and Nonlinear Radiation Memory
}

\author{
Thomas Mädler ${ }^{1} \dagger$ and Jeffrey Winicour ${ }^{2,3}$ \\ ${ }^{1}$ Núcleo de Astronomía, Facultad de Ingeniería, Universidad Diego Portales, Av. \\ Ejército 441, Santiago, Chile \\ ${ }^{2}$ Department of Physics and Astronomy \\ University of Pittsburgh, Pittsburgh, PA 15260, USA \\ 3 Max-Planck-Institut für Gravitationsphysik, Albert-Einstein-Institut, \\ 14476 Golm, Germany
}

\begin{abstract}
.
The Minkowski background intrinsic to the Kerr-Schild version of the Kerr metric provides a definition of a boosted spinning black hole. There are two Kerr-Schild versions corresponding to ingoing or outgoing principal null directions. The two corresponding Minkowski backgrounds and their associated boosts differ drastically. This has an important implication for the gravitational memory effect. A prior analysis of the transition of a non-spinning Schwarzschild black hole to a boosted state showed that the memory effect in the nonlinear regime agrees with the linearised result based upon the retarded Green function only if the final velocity corresponds to a boost symmetry of the ingoing Minkowski background. A boost with respect to the outgoing Minkowski background is inconsistent with the absence of ingoing radiation from past null infinity. We show that this results extends to the transition of a Kerr black hole to a boosted state and apply it to set upper and lower bounds for the boost memory effect resulting from the collision of two spinning black holes.

PACS numbers: 04.20.-q, 04.20.Cv, 04.20.Ex, 04.25.D-, 04.30-w
\end{abstract}

\section{Introduction}

The gravitational memory effect results in a net change in the relative separation of distant particles. After a wave passes it is determined by the difference between the initial and final radiation strain measured by a gravitational wave detector. The possibility of observable astrophysical consequences of the effect was first studied in linearised gravity where the memory resulted from a burst of massive particles which escaped to infinity, as described by the retarded solution of the linearised Einstein equations [1, 2]. In previous work [3], we showed that this result could also be obtained in linearised theory by considering the transition from an initial state whose exterior was described by a Schwarzschild metric at rest to a final state whose exterior was a boosted exterior Schwarzschild metric. This result was subsequently extended to the nonlinear

$\ddagger$ Email:thomas.maedler@mail.udp.cl 
treatment of the transition from a stationary to boosted Schwarzschild exterior [4, 5]. Here we further extend this treatment of the memory effect to the boosted Kerr metric.

Our linearised treatment of the memory effect was based upon the stationary and boosted versions of the ingoing Kerr-Schild version of the Schwarzschild metric to describe the far field of the initial and final states. In order to extend this result to the nonlinear case three major differences from the linearised theory had to be dealt with.

First, the linearised result was based upon the boost symmetry of the unperturbed Minkowski background. The Kerr-Schild metrics [6, 7] have the form

$$
g_{a b}=\eta_{a b}+2 H n_{a} n_{b}
$$

comprised of a Minkowski metric $\eta_{a b}$, a principal null vector $n_{a}$ (with respect to both $\eta_{a b}$ and $g_{a b}$ ) and a scalar field $H$. In the nonlinear case, the time reflection symmetry of the Schwarzschild metric leads to two different choices of a "Minkowski background" $\eta_{a b}$ depending on whether $n^{a}$ is chosen to be in the ingoing or outgoing direction. The relation between Boyer-Lindquist coordinates [8] and the ingoing and outgoing versions of Kerr-Schild coordinates is described in Sec. 2 .

Second, no analogue of the Green function exists in the nonlinear case to construct a retarded solution. Instead, the retarded solution due to the emission of radiation from an accelerated particle is characterised by the absence of ingoing radiation from past null infinity $\mathcal{I}^{-}$. The vanishing of radiation memory at $\mathcal{I}^{-}$is necessary for the absence of ingoing radiation. This condition allows the ingoing radiation strain, which forms the free characteristic initial data on $\mathcal{I}^{-}$, to be set to zero. Otherwise, non-zero radiation memory at $\mathcal{I}^{-}$would require ingoing radiation. Since an initial stationary Schwarzschild metric has vanishing radiation strain at $\mathcal{I}^{-}$, the final boosted metric must also have vanishing radiation strain at $\mathcal{I}^{-}$if there is no intervening ingoing radiation.

We found unexpected differences in the boosts associated with the ingoing and outgoing versions of the Kerr-Schild metric. The memory effect due to the final velocity of a Schwarzschild black hole is only correctly described by the boost $\mathcal{B}$ associated with the Poincaré group of the Minkowski background of the ingoing Kerr-Schild metric. This is because $\mathcal{B}$ belongs to the preferred Lorentz subgroup of the Bondi-Metzner-Sachs (BMS) [9] asymptotic symmetry group at $\mathcal{I}^{-}$which is picked out by the stationarity of the spacetime. This preferred Lorentz subgroup maps asymptotically shear-free crosssections of $\mathcal{I}^{-}$into themselves. Such cross-sections are non-distorted in the sense that their radiation strain vanishes. Thus $\mathcal{B}$ does not introduce strain at $\mathcal{I}^{-}$so that the transition from a stationary to boosted state is consistent with the lack of ingoing radiation from $\mathcal{I}^{-}$. But $\mathcal{B}$ is not a preferred BMS Lorentz symmetry of future null infinity $\mathcal{I}^{+}$where it induces a supertranslation component of the BMS group. As shown in [5], the strain introduced by this supertranslation results in non-zero radiation memory at $\mathcal{I}^{+}$. This memory effect is in precise agreement with the linearised result based upon the retarded Green function.

Conversely, the boost symmetry of the Minkowski metric associated with the 
outgoing version of the Kerr-Schild metric is a preferred (supertranslation-free) BMS symmetry at $\mathcal{I}^{+}$. Consequently, it introduces neither strain nor radiation memory at $\mathcal{I}^{+}$.

Third, in the nonlinear regime the mass of the final black hole depends upon the energy loss carried off by gravitational waves. This couples the memory effect due to the escape of an unbound particle to the Christodoulou memory effect [10] due to energy loss from gravitational radiation.

In extending this approach from the boosted Schwarzschild to the boosted Kerr metric there are further complications, in addition to the algebraic complexity. Unlike the Schwarzschild case, the principal null directions of the Kerr metric are not hypersurface orthogonal. As a result, there is no natural way to construct a null coordinate system in order to study the asymptotic behavior at null infinity. Here we show that there does exist a natural choice of hyperboloidal coordinates which provide a spacelike foliation extending asymptotically to null infinity. These hyperboloidal hypersurfaces are the null hypersurfaces of the Minkowski background for the KerrSchild version of the Kerr metric, which we abbreviate by KSK metric. For the ingoing KSK metric (see Sec. 3), the hyperboloids approach $\mathcal{I}^{-}$and for the outgoing case (see Sec. 4) they approach $\mathcal{I}^{+}$. The associated coordinates lead to a straightforward Penrose compactification of null infinity and allow an unambiguous treatment of the asymptotic radiation strain and identification of the Poincaré symmetries of the Minkowski background with BMS symmetries. Such curved space hyperboloidal hypersurfaces have been utilised in formulating the Cauchy problem for Einstein's equations in a manner suitable for radiation studies [11]. Their existence for the Kerr exterior was investigated in terms of an asymptotic series expansion in [12] and in terms of an implicit analytic scheme for the Teukolsky equation in [13. Here we construct a simple, geometrically natural and purely analytic hyperboloidal foliation that globally covers the entire Kerr exterior.

An additional factor is that the Kerr metric does not have the time reflection symmetry of the Schwarzschild metric, but instead a $(\tau, \varphi) \rightarrow(-\tau,-\varphi)$ symmetry in Boyer-Lindquist coordinates, as described in Sec. 2, This further complicates relating asymptotic properties at $\mathcal{I}^{-}$and $\mathcal{I}^{+}$.

Although these issues introduce considerable technical complication, our main results for the Schwarzschild case extend without change to the Kerr case. This could be expected on heuristic grounds since the results depend only upon the asymptotic behavior of the boosted metric for which the spin of the black hole enters at a higher order in $1 / r$ than its mass. Nevertheless, an important aspect of the memory effect is the supertranslation ambiguity, which enters the bookkeeping of angular momentum. At some deeper level, which deserves further investigation, the spin of the black hole should enter.

There has been extensive elaboration and generalisation of the memory effect since its first astrophysically relevant discussion [1] in terms of the linearised description of the burst of massive particles ejected to infinity. This remained the dominant mechanism 
until Christodoulou demonstrated a purely nonlinear memory effect due to mass loss from an isolated system by gravitational waves. Extensive subsequent work showed that the Christodoulou effect was not necessarily a nonlinear effect but that analogous effects occurred in linear theory due to radiative mass loss to null infinity by Maxwell fields or other rest mass zero fields or particles. This motivated a change in terminology of the Christodoulou effect to a "null" memory effect [14], as opposed to a nonlinear memory effect. It is now understood that the memory effect results either by the radiation to null infinity by zero rest mass fields (or particles) or by transport to timelike infinity by massive particles boosted with escape velocity.

Three distinct mechanisms for the memory effect have been proposed. The first is what historically has been called the "linear" memory effect resulting from a burst of ejected particles, as described in [1]. For the present purpose, we refer this as the boost mechanism. The second mechanism, i.e. null memory, generalises the Christodoulou effect to rest mass zero fields or particles [15, 16, 17, 18,. The third is a homogeneous wave mechanism [3] due to source free graviational waves emanating from $\mathcal{I}^{-}$to $\mathcal{I}^{+}$. All three mechanisms exist in linearised theory. They can be separated into an "ordinary" memory effect in which no energy is lost to $\mathcal{I}^{-}$and a null memory effect. The treatment of null memory only depends upon asymptotic propertiesin the neighborhood of null infinity, which readily extends to nonlinear gravitational waves, as demonstrated in terms of the asymptotic properties of the vacuum Bianchi identities [19], or in terms of the asymptotic properties of Maxwell or other mass zero radiation fields propagating in curved spacetime. These mechanisms combine to form the net memory effect measured by the change in radiation strain between the infinite future and infinite past retarded times.

In this paper we demonstrate how the memory effect due to a boosted Kerr black hole can be treated in the nonlinear case. The nonlinear treatment is purely asymptotic but, as explained above, differs considerably from the linearised derivation based upon the retarded flat space Green function. No linear approximations are involved.

Supposedly the mechanism for the homogeneous wave memory also extends to the nonlinear theory although there is no general proof by construction of exact solutions as in the linearised case. Other memory effects based upon changes in angular momentum or center-of-mass integrals have also been proposed [20, 21, 22].

Although an exact Kerr metric is an unrealistic approximation to the exterior of a dynamical spacetime, it is a reasonable far field approximation for the final black hole state, in accordance with the no hair scenario. In Sec. 5, we derive the nonlinear memory effect for the transition from a stationary to boosted Kerr black hole. In Sec. 6, we show how this result may be generalised and, as an example, treat the collision of two boosted black holes to form a final black hole. We derive upper and lower bounds for the boost memory in terms of the final mass of the Kerr black hole resulting from the collision. Here the mass of the final black hole depends upon the energy loss in the intervening radiative period, which leads to the null memory effect. The difference between the final and initial radiation strains, as measured by the net memory effect, 
combines both the null and boost effects in a way which can only be determined by knowledge of the intervening radiative period. This is discussed further in Sec. 6.

Kerr-Schild metrics have played an important role in the construction of exact solutions [23]. Because their metric form (11) is invariant under the Lorentz symmetry of the Minkowski background metric $\eta_{a b}$, the boosted KSK metric has been important in numerical relativity in prescribing initial data for superimposed boosted and spinning black holes in a binary orbit [24, 25]. The initial data for numerical simulations are prescribed in terms of the ingoing version of the KSK metric, whose advanced time coordinatisation extends across the future event horizon. The initial black hole velocities are generated by the boost symmetry of the Minkowski background for the ingoing KSK metric. This is in accord with our treatment of radiation memory.

We denote abstract spacetime indices by $a, b, \ldots$ and coordinate indices by $\alpha, \beta, \ldots$. In addition, we denote 3-dimensional spatial indices for the background inertial coordinates by $i, j, \ldots$, and denote the associated 2-dimensional spherical coordinates by $x^{A}=(\theta, \phi)$. We often use the standard comma notation to denote partial derivatives, e.g. $f_{, \alpha}=\partial f / \partial x^{\alpha}$.

The distinction between the ingoing and outgoing Kerr-Schild metrics and their associated background Minkowski symmetries requires extra notational care. We retain the notation in our previous papers in which a superscript $(+)$ denotes quantities associated with the advanced time versions of the Schwarzschild metric and a superscript $(-)$ denotes quantities associated with the retarded time version. Corresponding to this notation, we use a superscript $(+)$ for quantities associated with the ingoing version of the KSK metric and a superscript (-) for quantities associated with the outgoing version. As an example, the ingoing principal null vector is denoted by $n_{a}^{(+)}$and its null rays emanate from past null infinity $\mathcal{I}^{-}$and extend across the future event horizon; and

the outgoing principal null vector is denoted by $n_{a}^{(-)}$, whose null rays extend to future null infinity $\mathcal{I}^{+}$. Because the memory effect is gauge invariant, it can be computed in either in the inertial coordinates $x^{(+) \alpha}$ or $x^{(-) \alpha}$ associated with the ingoing or outgoing Minkowski backgrounds of the Kerr-Schild metric, respectively. In Sec. 5, for technical convenience we choose the ingoing version $x^{(+) \alpha}$. However, the relation to the outgoing version $x^{(-) \alpha}$ is necessary to compute limits at $\mathcal{I}^{+}$. The details of this transformation are presented in Sec. 2 .

\section{The Kerr-Schild Kerr (KSK) metric and its associated Minkowski backgrounds}

The Boyer-Lindquist coordinates [8], which we denote by $(\tau, r, \vartheta, \varphi)$, provide the intermediate connection between the ingoing and outgoing versions of the KSK metric. In these coordinates, the Kerr metric is

$$
d s^{2}=-d \tau^{2}+\Sigma\left(\frac{d r^{2}}{\Delta}+d \vartheta^{2}\right)+\left(r^{2}+a^{2}\right) \sin ^{2} \vartheta d \varphi^{2}+\frac{2 m r}{\Sigma}\left(a \sin ^{2} \vartheta d \varphi-d \tau\right)^{2},(2)
$$


where $m$ is the mass, $a$ is the specific angular momentum and

$$
\Sigma=r^{2}+a^{2} \cos ^{2} \vartheta, \quad \Delta=r^{2}-2 m r+a^{2} .
$$

Note that this substitutes $a \rightarrow-a$ in the formulae of [6, 7] to agree with the standard convention that the sense of rotation is in the positive $\varphi$ direction.

The KSK metric can be expressed in terms of either the ingoing principal null direction $n^{(+) a}$ or the outgoing principal null direction $n^{(-) a}$. These two forms of the metric have different inertial coordinates $x^{( \pm) \alpha}=\left(t^{( \pm)}, x^{( \pm)}, y^{( \pm)}, z^{( \pm)}\right)$for their corresponding Minkowski backgrounds $\eta_{a b}^{( \pm)}$. The main details have been worked out by considering the $(\tau, \varphi) \rightarrow(-\tau,-\varphi)$ reflection symmetry of the Kerr metric in Boyer-Lindquist coordinates [8]. The coordinate transformations leading from (2) to the ingoing $(+)$ or outgoing $(-)$ Kerr-Schild form involve a generalisation of the Schwarzschild tortoise coordinate $r^{*}$,

$$
\begin{aligned}
r^{*} & =\int\left(\frac{r^{2}+a^{2}}{r^{2}-2 m r+a^{2}}\right) d r \\
& =r+m \ln \left(\frac{r^{2}-2 m r+a^{2}}{4 m^{2}}\right)+\frac{m^{2}}{\sqrt{m^{2}-a^{2}}} \ln \left(\frac{r-m-\sqrt{m^{2}-a^{2}}}{r-m+\sqrt{m^{2}-a^{2}}}\right)
\end{aligned}
$$

and the intermediate angles

$$
\Phi^{ \pm}=\varphi \pm a \int \frac{d r}{\Delta}=\varphi \pm \frac{a}{2 \sqrt{m^{2}-a^{2}}} \ln \left(\frac{r-m-\sqrt{m^{2}-a^{2}}}{r-m+\sqrt{m^{2}-a^{2}}}\right) .
$$

The transformation from Boyer-Lindquist to Kerr-Schild coordinates can then be written compactly as

$$
\begin{aligned}
t^{( \pm)} & =\tau \pm\left(r-r^{*}\right) \\
x^{( \pm)}+i y^{( \pm)} & =\sqrt{r^{2}+a^{2}} \sin \vartheta \exp \left\{\mathrm{i}\left[\Phi^{( \pm)} \pm \arctan (a / r)\right]\right\} \\
z^{( \pm)} & =r \cos \vartheta .
\end{aligned}
$$

Here $x^{(+) 2}+y^{(+) 2}=x^{(-) 2}+y^{(-) 2}$ so we simply denote $x^{2}+y^{2}=x^{( \pm) 2}+y^{( \pm) 2}$. Similarly, we denote $z=z^{( \pm)}$. The Boyer-Lindquist radial coordinate $r$ is then determined implicitly by

$$
\frac{x^{2}+y^{2}}{r^{2}+a^{2}}+\frac{z^{2}}{r^{2}}=1
$$

independent of the choice of background coordinates.

The resulting Kerr Schild metrics in the background inertial coordinates $x^{( \pm) \alpha}$ are

$$
g_{\alpha \beta}=\eta_{\alpha \beta}^{( \pm)}+2 H n_{\alpha}^{( \pm)} n_{\beta}^{( \pm)}, H=\frac{m r^{3}}{r^{4}+a^{2} z^{2}},
$$

where the ingoing and outgoing versions of the principal null vectors have components

$n_{\alpha}^{(+)}\left(x^{(+) \beta}\right)=\left(n_{t}^{(+)}, n_{x}^{(+)}, n_{y}^{(+)}, n_{z}^{(+)}\right)=\left(-1,-\frac{r x^{(+)}+a y^{(+)}}{r^{2}+a^{2}},-\frac{r y^{(+)}-a x^{(+)}}{r^{2}+a^{2}},-\frac{z}{r}\right)$,

and

$n_{\alpha}^{(-)}\left(x^{(-) \beta}\right)=\left(n_{t}^{(-)}, n_{x}^{(-)}, n_{y}^{(-)}, n_{z}^{(-)}\right)=\left(-1, \frac{r x^{(-)}-a y^{(-)}}{r^{2}+a^{2}}, \frac{r y^{(-)}+a x^{(-)}}{r^{2}+a^{2}}, \frac{z}{r}\right)$. 
Recall that $\operatorname{det}\left(g_{\alpha \beta}\right)=\operatorname{det}\left(\eta_{\alpha \beta}^{ \pm}\right)=-1$ for Kerr-Schild metrics in these background coordinates.

From (6) and (7), it follows that the time coordinates of the two Minkowski backgrounds are related by

$$
t^{(+)}=t^{(-)}+2\left(r-r^{*}\right)
$$

and the spatial coordinates are related by

$$
x^{(+)}+i y^{(+)}=\left(x^{(-)}+i y^{(-)}\right) e^{i \Psi(r)}, \quad z=z^{(+)}=z^{(-)},
$$

where

$$
\Psi(r):=\frac{a}{\sqrt{m^{2}-a^{2}}} \ln \left(\frac{r-m-\sqrt{m^{2}-a^{2}}}{r-m+\sqrt{m^{2}-a^{2}}}\right)+2 \arctan (a / r) .
$$

We set

$$
\rho^{2}=x^{2}+y^{2}+z^{2},
$$

and introduce the standard spherical coordinates $\left(\rho, \theta, \phi^{( \pm)}\right)$for the Minkowski backgrounds,

$$
x^{( \pm)}+i y^{( \pm)}=\rho \sin \theta \exp \left[i \phi^{( \pm)}\right] . \quad z=\rho \cos \theta .
$$

Here $\rho$ and $\theta$, but not $\phi^{( \pm)}$, are background independent.

\section{3. $\mathcal{I}^{-}$and the boost symmetry}

In [4, we showed that the linearised memory effect arising from the retarded solution for a freely ejected particle could be obtained from the boosted version of the advanced time Kerr-Schild-Schwarzschild metric. In that treatment, the boost was a Lorentz symmetry of the linearised Minkowski background. However, this could not be extended unambiguously to the nonlinear case, where there are two different choices of boost symmetry corresponding to the Minkowski backgrounds $\eta_{\alpha \beta}^{(+)}$or $\eta_{\alpha \beta}^{(-)}$of the ingoing or outgoing versions of the curved space Kerr-Schild-Schwarzschild metric. In the curved space case, it is not the choice of ingoing or outgoing Kerr-Schild metric (which are algebraically equal) but the choice of boost that leads to the essential result. In particular, because the boost symmetry of the ingoing background $\eta_{\alpha \beta}^{(+)}$is a preferred BMS symmetry of $\mathcal{I}^{-}$it does not produce ingoing radiation strain at $\mathcal{I}^{-}$but it does induce a supertranslation at $\mathcal{I}^{+}$, which leads to outgoing radiation strain.

In the Schwarzschild case, the null hypersurfaces determined by the principal null directions provide a simple approach to construct null infinity. In the Kerr case, this is more complicated because the principal null directions are not hypersurface orthogonal. For this reason, we describe $\mathcal{I}^{-}$in the Kerr case by considering the null spherical coordinates associated with the Minkowski background $\eta_{\alpha \beta}^{(+)}$,

$$
\tilde{x}^{(+) \alpha}=\left(v, \rho, \theta, \phi^{(+)}\right), \quad v=t^{(+)}+\rho .
$$

In these coordinates,

$$
\eta_{\alpha \beta}^{(+)} d \tilde{x}^{(+) \alpha} d \tilde{x}^{(+) \beta}=-d v^{2}+2 d v d \rho+\rho^{2} d q^{(+) 2},
$$


where $d q^{(+) 2}=q_{A B}^{(+)} d x^{(+) A} d x^{(+) B}=d \theta^{2}+\sin ^{2} \theta d \phi^{(+) 2}$ is the unit sphere metric. The ingoing KSK metric takes the form

$$
g_{\alpha \beta} d \tilde{x}^{(+) \alpha} d \tilde{x}^{(+) \alpha}=-d v^{2}+2 d v d \rho+\rho^{2} d q^{(+) 2}+2 H\left(n_{\alpha}^{(+)} d \tilde{x}^{(+) \alpha}\right)^{2},
$$

where, using (9) and (11),

$$
n_{\alpha}^{(+)} d \tilde{x}^{(+) \alpha}=-d v+\left(1-\frac{r}{\rho}\right) d \rho+\frac{a \rho^{2} \sin \theta}{r\left(r^{2}+a^{2}\right)}\left(a \cos \theta d \theta+r \sin \theta d \phi^{(+)}\right) .
$$

The inverse property of Kerr-Schild metrics,

$$
g^{a b}=\eta^{(+) a b}-2 H n^{(+) a} n^{(+) b},
$$

implies

$$
g^{a b}\left(\partial_{a} v\right) \partial_{b} v=-2 H\left(n^{(+) a} \partial_{a} v\right)^{2} .
$$

As a result, since $H \geq 0$, the hypersurfaces $v=$ const are spacelike except in the limiting Schwarzschild case, where $a=0$ and the hypersurfaces are null. Explicitly, (11) leads to

$$
n^{(+) \alpha} \partial_{\alpha} v=1-\frac{r \rho}{r^{2}+a^{2}}-\frac{a^{2} z^{2}}{r \rho\left(r^{2}+a^{2}\right)},
$$

or, using (9),

$$
n^{(+) \alpha} \partial_{\alpha} v=1-\frac{r}{\rho} .
$$

\subsection{Compactification of past null infinity}

For $a \neq 0$, the hypersurfaces $v=$ const are spacelike hyperboloids in the Kerr geometry which approach $\mathcal{I}^{-}$asymptotically. In order to compactify $\mathcal{I}^{-}$, we replace the hyperboloidal spherical coordinates $\tilde{x}^{(+) \alpha}=\left(v, \rho, \theta, \phi^{(+)}\right)$by the compactified

coordinates $\hat{x}^{(+) \alpha}=\left(v, \ell, \theta, \phi^{(+)}\right)$, where $\ell=1 / \rho$. In these coordinates $\mathcal{I}^{-}$is given by $\ell=0$.

Now introduce the conformally rescaled metric denoted by $\hat{g}_{a b}=\ell^{2} g_{a b}$. The conformal metric is given in the compactified coordinates by

$$
\hat{g}_{\alpha \beta} d \hat{x}^{(+) \alpha} d \hat{x}^{(+) \beta}=-\ell^{2} d v^{2}-2 d v d \ell+d q^{(+) 2}+2 H\left(\ell n_{\alpha}^{(+)} d \hat{x}^{\alpha(+)}\right)^{2},
$$

with

$$
\ell n_{\alpha}^{(+)} d \hat{x}^{(+) \alpha}=-\ell d v-\left[\ell^{-1}-r\right] d \ell+\frac{a \sin \theta}{r \ell\left(r^{2}+a^{2}\right)}\left(a \cos \theta d \theta+r \sin \theta d \phi^{(+)}\right) .
$$

The asymptotic behaviour of $\hat{g}_{\alpha \beta}$ depends upon the asymptotic expansion of the Boyer-Lindquist coordinate $r$. From (9),$r$ is determined by the quartic equation

$$
r^{4}-\left(x^{2}+y^{2}+z^{2}-a^{2}\right) r^{2}-a^{2} z^{2}=r^{4}-\left(\rho^{2}-a^{2}\right) r^{2}-a^{2} \rho^{2} \cos ^{2} \theta=0 .
$$

The solution

$$
\begin{aligned}
r & =\frac{1}{2} \sqrt{2 \rho^{2}-2 a^{2}+2 \sqrt{\rho^{4}-2 \rho^{2} a^{2}+a^{4}+4 a^{2} z^{2}}} \\
& =\frac{1}{2} \sqrt{2 \rho^{2}-2 a^{2}+2 \sqrt{\rho^{4}-2 \rho^{2} a^{2}+a^{4}+4 a^{2} \rho^{2} \cos ^{2} \theta}}
\end{aligned}
$$


has the asymptotic $\ell$ expansion about $\mathcal{I}^{-}$

$$
r(\ell, \theta)=\ell^{-1}-\frac{a^{2} \sin ^{2} \theta}{2} \ell-\frac{a^{4} \sin ^{2} \theta\left(1-5 \cos ^{2} \theta\right)}{8} \ell^{3}+O\left(\ell^{5}\right), \quad \ell=1 / \rho .
$$

As a result, $H, \ell n_{\alpha}^{(+)} d \hat{x}^{(+) \alpha}$ and the conformal metric have the asymptotic $\ell$-expansions

$$
\begin{aligned}
H=m \ell[1+ & \left.\frac{a^{2}}{2}\left(1-3 \cos ^{2} \theta\right) \ell^{2}+\frac{a^{4}}{8}\left(3-30 \cos ^{2} \theta+35 \cos ^{4} \theta\right) \ell^{4}+O\left(\ell^{6}\right)\right],(32) \\
\ell n_{\alpha}^{(+)} d \hat{x}^{\alpha}= & \ell\left(-d v-\frac{a^{2}}{2} \sin ^{2} \theta d \ell+a \sin ^{2} \theta d \phi^{(+)}\right)+\ell^{2}\left(a^{2} \sin \theta \cos \theta d \theta\right) \\
& +\ell^{3}\left[\frac{a^{4}}{8}\left(5 \cos ^{2} \theta-1\right) \sin ^{2} \theta d \ell-a^{3} \sin ^{2} \theta \cos ^{2} \theta d \phi^{(+)}\right] \\
& +\ell^{4}\left[\frac{a^{4}}{2}\left(1-3 \cos ^{2} \theta\right) \sin \theta \cos \theta d \theta\right]+O\left(\ell^{5}\right)
\end{aligned}
$$

and

$$
\begin{gathered}
\hat{g}_{\alpha \beta} d \hat{x}^{(+) \alpha} d \hat{x}^{(+) \beta}=-2 d v d \ell+d q^{(+) 2}-\ell^{2} d v^{2}+2 m \ell^{3}\left(d v+\frac{a^{2}}{2} \sin ^{2} \theta d \ell-a \sin ^{2} \theta d \phi^{(+)}\right)^{2} \\
+\ell^{4}\left[-4 m a^{2}\left(d v+\frac{a^{2}}{2} \sin ^{2} \theta d \ell-a \sin ^{2} \theta d \phi^{(+)}\right) \sin \theta \cos \theta d \theta\right]+O\left(\ell^{5}\right)
\end{gathered}
$$

with the determinant $\hat{g}=-\sin ^{2} \theta$ and inverse

$$
\begin{aligned}
& \hat{g}^{\alpha \beta} \partial_{\hat{x}^{(+) \alpha}} \partial_{\hat{x}^{(+) \beta}}=-2 \partial_{v} \partial_{\ell}+q^{(+) A B} \partial_{\hat{x}^{(+) A}} \partial_{\hat{x}^{(+) B}}+\ell^{2} \partial_{\ell} \partial_{\ell}-2 m \ell^{3}\left(\frac{a^{2} \sin ^{2} \theta}{2} \partial_{v}+\partial_{\ell}+a \partial_{\phi}(+)\right)^{2} \\
& -4 a^{2} m \ell^{4}\left[\left(\frac{a^{2} \sin ^{2} \theta}{2} \partial_{v}+\partial_{\ell}+a \partial_{\phi^{(+)}}\right) \sin \theta \cos \theta\right] \partial_{\theta}+O\left(\ell^{5}\right)
\end{aligned}
$$

As a result, $\mathcal{I}^{-}$, given by $\ell=0$ with $\left.\hat{\nabla}_{a} \ell\right|_{\mathcal{I}^{-}} \neq 0$, has Penrose compactification with metric

$$
\left.\hat{g}_{\alpha \beta} d \hat{x}^{(+) \alpha} d \hat{x}^{(+) \beta}\right|_{\mathcal{I}^{-}}=-2 d v d \ell+d q^{(+) 2},
$$

i.e. $\mathcal{I}^{-}$is a null hypersurface with standard asymptotically Minkowskian geometry consisting of unit sphere cross-sections. In addition, it is straightforward to verify that

$$
\left.\hat{\nabla}_{\alpha} \hat{\nabla}_{\beta} \ell\right|_{\mathcal{I}^{-}}=0
$$

so that $\ell$ is a conformal factor in which the shear and divergence of $\mathcal{I}^{-}$vanish. Thus $\ell$ is a preferred conformal factor for which the compactification of $\mathcal{I}^{-}$has the same asymptotic properties as described by a conformal Bondi frame [29]. This allows a simple description of the BMS asymptotic symmetries and other physical properties of $\mathcal{I}^{-}$.

\subsection{Physical properties of $\mathcal{I}^{-}$}

The Lorentz symmetries of $\eta_{\alpha \beta}^{(+)}$are not symmetries of the KSK metric but they are preferred BMS symmetries of $\mathcal{I}^{-}$. The remaining BMS symmetries are the supertranslations on $\mathcal{I}^{-}, v \rightarrow v+\alpha\left(\theta, \phi^{(+)}\right)$. The supertranslations with $\alpha$ composed 
of $l=0$ and $l=1$ spherical harmonics correspond to the Poincare translations of the Minkowski background.

In the hyperboloidal coordinates $\left(v, \ell, x^{(+) A}\right)$, where $x^{(+) A}=\left(\theta, \phi^{(+)}\right)$, the strain tensor $\sigma_{A B}\left(v, x^{(+) A}\right)$ describing the ingoing radiation from $\mathcal{I}^{-}$is determined by the asymptotic expansion of the metric according to

$$
\hat{g}_{A B}=q_{A B}+2 \ell \sigma_{A B}+O\left(\ell^{2}\right) .
$$

Here $\sigma_{A B}\left(v, x^{(+) C}\right)$ is trace-free and corresponds to the asymptotic shear of the ingoing null hypersurfaces emanating from the $v=$ const cross-sections of $\mathcal{I}^{-}$or, equivalently, the radiation strain of the cross-sections. It can be described by the spin-weight-2 function

$$
\sigma\left(v, x^{(+) A}\right)=q^{A} q^{B} \sigma_{A B}\left(v, x^{(+) A}\right),
$$

where $q^{A}$ is the complex polarisation dyad associated with the unit sphere metric on $\mathcal{I}^{-}$,

$$
q_{A B}^{(+)}=\frac{1}{2}\left(q_{A} \bar{q}_{B}+\bar{q}_{A} q_{B}\right), \quad q^{A} \bar{q}_{A}=2, \quad q^{A} q_{A}=0, \quad q_{A}=q_{A B}^{(+)} q^{B} .
$$

For the standard choice of spherical coordinates, we set $q^{A} \partial_{(+) A}=\partial_{\theta}+(i / \sin \theta) \partial_{\phi^{(+)}}$. This normalisation implies

$$
\sigma=\sigma_{\theta \theta}-\frac{\sigma_{\phi^{(+)} \phi^{(+)}}}{\sin ^{2} \theta}+\frac{2 i \sigma_{\theta \phi}(+)}{\sin \theta}
$$

which corresponds to the standard plus/cross decomposition, as used in [3, 4, 26]. The normalisation used in [5] inadvertently reduced $\sigma$ by a factor of $1 / 2$.

In terms of the physical space description in the associated inertial Cartesian coordinates, the polarisation dyad $q^{A}$ has components $q^{i}=\rho Q^{i}$ where

$$
Q^{i}=\frac{1}{\rho} x^{i}{ }_{(+) A} q^{A}=\left(\cos \theta \cos \phi^{(+)}-i \sin \phi^{(+)}, \cos \theta \sin \phi^{(+)}+i \cos \phi^{(+)},-\sin \theta\right),
$$

where $Q_{i} \bar{Q}^{i}=2, Q_{i} Q^{i}=0$ and $Q_{i} x^{i}=0$. Here we raise and lower the Cartesian indices $i, j, \ldots$ for fields in the Euclidean background according to the example $Q_{i}=\delta_{i j} Q^{j}$. Then $\sigma$ is determined by the physical space metric according to

$$
\sigma\left(v, x^{(+) A}\right)=\lim _{\rho \rightarrow \infty} \frac{\rho}{2} Q^{i} Q^{j} g_{i j},
$$

where the limit at $\mathcal{I}^{-}$is taken holding $\left(v, x^{(+) A}\right)$ constant. For the unboosted KSK metric,

$$
\sigma\left(v, x^{(+) A}\right)=\lim _{\rho \rightarrow \infty} \rho H\left(Q^{i} n_{i}^{(+)}\right)^{2}=\lim _{\rho \rightarrow \infty} m\left(Q^{i} n_{i}^{(+)}\right)^{2} .
$$

A straightforward calculation gives

$$
Q^{i} n_{i}^{(+)}=\frac{\rho a \sin \theta(a \cos \theta+i r)}{r\left(r^{2}+a^{2}\right)} .
$$

With reference to (31), it follows that $Q^{i} n_{i}^{(+)}=O(1 / \rho)$ so that the radiation strain vanishes at the advanced times $v=$ const picked out by the null cones of $\eta_{\alpha \beta}^{(+)}$. However, 
under the supertranslation $v \rightarrow v+\alpha\left(x^{(+) A}\right)$ the radiation strain has the gauge freedom (cf. [26])

$$
\sigma \rightarrow \sigma+q^{A} q^{B} \varlimsup_{(+) A} \widetilde{\partial}_{(+) B} \alpha\left(x^{(+) A}\right)
$$

where $\widetilde{\partial}_{(+) A}$ is the covariant derivative with respect to the unit sphere metric $d q^{(+) 2}$. As a result, even in a stationary epoch where $\sigma\left(v, x^{(+) A}\right)=\sigma\left(x^{(+) A}\right)$, cross-sections of $\mathcal{I}^{-}$ distorted by a supertranslation have non-vanishing strain.

\subsection{The boost symmetry of $\mathcal{I}^{-}$}

The boost symmetries $\mathcal{B}$ of the Minkowski background $\eta_{a b}^{(+)}$are not exact symmetries of the Kerr metric but they are asymptotic BMS symmetries of $\mathcal{I}^{-}$which are preferred in the sense that they map strain-free cross-sections into strain-free cross-sections. It is this asymptotic property of $\mathcal{B}$ which is essential for our treatment of the memory effect. However, the BMS transformations are only uniquely determined to first order in $\ell$ by the requirement that they be asymptotic symmetries. In the compactified coordinates $\hat{x}^{(+) \alpha}=\left(v, \ell, \theta, \phi^{(+)}\right)$, this leads to an equivalence class of BMS transformations in which the identity takes the form [27]

$$
\hat{x}^{(+) \alpha} \rightarrow \hat{x}^{(+) \alpha}+O\left(\ell^{2}\right) .
$$

Instead of the Minkowski boost symmetries $\mathcal{B}$ we could use any equivalent BMS subgroup in our treatment of the memory effect. The resulting $O\left(\ell^{2}\right)$ terms in the boost would not affect the radiation strain at $\mathcal{I}^{-}$or $\mathcal{I}^{+}$.

Consider now a Minkowski boost $\mathcal{B}$ whose 4 -velocity has components $v^{\alpha}=\Gamma\left(1, V^{i}\right)$, where $V^{i}=V l^{i}$, with direction cosines $l^{i}$, and $\Gamma=1 / \sqrt{1-V^{2}}$. Under this boost, $\eta_{\alpha \beta}^{(+)} \rightarrow \eta_{\alpha \beta}^{(+)}$. The boosted coordinates $x_{\mathcal{B}}^{(+) \alpha}$ are given by the Lorentz transformation

$$
x_{\mathcal{B}}^{(+) \alpha}=\Lambda_{\beta}^{\alpha} x^{(+) \beta},
$$

where

$$
\begin{aligned}
& \Lambda^{t}{ }_{t}=\Gamma, \\
& \Lambda^{t}{ }_{i}=\Lambda^{i}{ }_{t}=-\Gamma V_{i}, \\
& \Lambda^{i}{ }_{j}=\delta^{i}{ }_{j}+(\Gamma-1) l^{i} l_{j} .
\end{aligned}
$$

The boosted coordinates are

$$
\begin{aligned}
t_{\mathcal{B}}^{(+)} & =\Gamma\left(t^{(+)}-V_{i} x^{(+) i}\right), \\
x_{\mathcal{B}}^{(+) i} & =x^{(+) i}+\left[-\Gamma V t^{(+)}+(\Gamma-1) l_{j} x^{(+) j}\right] l^{i},
\end{aligned}
$$

e.g, for a boost in the $z$-direction, $l^{x}=l^{y}=l^{z}-1=0, x_{\mathcal{B}}^{(+)}=x^{(+)}, y_{\mathcal{B}}^{(+)}=y^{(+)}$and

$$
\begin{aligned}
& t_{B}^{(+)}=\Gamma\left(t^{(+)}-V z^{(+)}\right), \\
& z_{\mathcal{B}}^{(+)}=\Gamma\left(z^{(+)}-V t^{(+)}\right) .
\end{aligned}
$$

The background spherical radius $\rho$ transforms as

$$
\rho^{2} \rightarrow \rho_{\mathcal{B}}^{2}=x^{(+) \alpha} x_{\alpha}^{(+)}+\left(v_{\alpha} x^{(+) \alpha}\right)^{2}=-\left[t^{(+)}\right]^{2}+\rho^{2}+\Gamma^{2}\left(t^{(+)}-V_{i} x^{(+) i}\right)^{2} .
$$


Setting $t^{(+)}=v-\rho$, the large $\rho$ expansion of $\rho_{\mathcal{B}}$ about $\mathcal{I}^{-}$holding $v$ constant is

$$
\rho_{\mathcal{B}}=\rho \Gamma\left(1+V_{i} \rho^{i}\right)-\frac{v V \Gamma\left(V+l_{i} \rho^{i}\right)}{1+V_{i} \rho^{i}}+O(1 / \rho)
$$

where

$$
\rho^{i}=x^{(+) i} / \rho=\left(\sin \theta \cos \phi^{(+)}, \sin \theta \sin \phi^{(+)}, \cos \theta\right) .
$$

The boosted version of the Boyer-Lindquist radial coordinate (29),

$$
r_{\mathcal{B}}=r\left(\rho_{\mathcal{B}}, z_{\mathcal{B}}\right)=\frac{1}{2} \sqrt{2 \rho_{\mathcal{B}}^{2}-2 a^{2}+2 \sqrt{\rho_{\mathcal{B}}^{4}-2 \rho_{\mathcal{B}}^{2} a^{2}+a^{4}+4 a^{2} z_{\mathcal{B}}^{2}}}
$$

has the large $\rho$ expansion about $\mathcal{I}^{-}$, holding $v=t^{(+)}+\rho$ constant,

$$
r_{\mathcal{B}}=\left(1+V_{i} \rho^{i}\right) \Gamma \rho-\frac{v V \Gamma\left(V+l_{i} \rho^{i}\right)}{1+V_{i} \rho^{i}}+O(1 / \rho)=\rho_{\mathcal{B}}+O(1 / \rho) .
$$

This leads to the expansion of the boosted version of the Kerr-Schild function about $\mathcal{I}^{-}$,

$$
H_{\mathcal{B}}=\frac{m r_{\mathcal{B}}^{3}}{r_{\mathcal{B}}^{4}+a^{2} z_{\mathcal{B}}^{2}}=\frac{m}{\left(1+V_{i} \rho^{i}\right) \Gamma \rho}\left[1+\frac{V\left(V+l_{i} \rho^{i}\right) v}{\left(1+V_{i} \rho^{i}\right)^{2} \rho}\right]+O\left(1 / \rho^{2}\right) .
$$

It follows from (11) and (31) that the ingoing principle null direction has asymptotic behaviour

$$
n_{\alpha}^{(+)}=-\nabla_{\alpha}\left(t^{(+)}+\rho\right)+O(1 / \rho) .
$$

Using the covariant substitutions $-\nabla_{\alpha} t \rightarrow v_{\alpha}$ and $\nabla_{\alpha} \rho \rightarrow\left[x_{\alpha}+v_{\beta} x^{(+) \beta} v_{\alpha}\right] / \rho_{\mathcal{B}}$, its boosted version $N_{\alpha}:=n_{\mathcal{B} \alpha}^{(+)}$has asymptotic behaviour

$$
N_{\alpha}=v_{\alpha}-\frac{1}{\rho_{\mathcal{B}}}\left(x_{\alpha}^{(+)}+v_{\beta} x^{(+) \beta} v_{\alpha}\right)+O(1 / \rho) .
$$

Setting $t^{(+)}=v-\rho$, the expansion of the boosted version of (45) about $\mathcal{I}^{-}$, holding $v$ constant, then leads to

$$
Q^{i} N_{i}=\left(1-\frac{1}{\rho_{\mathcal{B}}} v_{\beta} x^{(+) \beta}\right) Q^{i} v_{i}+O(1 / \rho)=\left(1-\frac{1}{\rho_{\mathcal{B}}} \rho \Gamma\left(1+V_{i} \rho^{i}\right)\right) Q^{i} v_{i}+O(1 / \rho),
$$

so it follows from (57) that $Q^{i} N_{i}=O(1 / \rho)$.

Thus, referring to the boosted version of (44),

$$
\sigma_{\mathcal{B}}\left(v, x^{(+) A}\right)=\lim _{\rho \rightarrow \infty} \rho H_{\mathcal{B}}\left(Q^{i} N_{i}\right)^{2}=0
$$

i.e. the strain at $\mathcal{I}^{-}$vanishes for the boosted KSK metric, as expected since the boost is a preferred BMS symmetry of $\mathcal{I}^{-}$. 


\section{Future null infinity}

Following the procedure for treating $\mathcal{I}^{-}$, we describe $\mathcal{I}^{+}$in terms of the KSK metric by considering retarded null spherical coordinates associated with the Minkowski background, $\eta_{a b}^{(-)}$,

$$
\tilde{x}^{(-) \alpha}=\left(u, \rho, \theta, \phi^{(-)}\right), \quad u=t^{(-)}-\rho .
$$

In these coordinates,

$$
\eta_{\alpha \beta}^{(-)} d \tilde{x}^{(-) \alpha} d \tilde{x}^{(-) \beta}=-d u^{2}-2 d u d \rho+\rho^{2} d q^{(-) 2},
$$

where $d q^{(-) 2}=q_{A B}^{(-)} d x^{(-) A} d x^{(-) B}=d \theta^{2}+\sin ^{2} \theta d \phi^{(-) 2}$ and the outgoing version of the KSK metric has components

$$
g_{\alpha \beta} d \tilde{x}^{(-) \alpha} d \tilde{x}^{(-) \beta}=-d u^{2}-2 d u d \rho+\rho^{2} d q^{(-) 2}+2 H\left(n_{\alpha}^{(-)} d \tilde{x}^{(-) \alpha}\right)^{2} .
$$

The inverse form of the outgoing KSK metric,

$$
g^{a b}=\eta^{(-) a b}-2 H n^{(-) a} n^{(-) b},
$$

now implies

$$
g^{a b}\left(\partial_{a} u\right) \partial_{b} u=-2 H\left(n^{(-) a} \partial_{a} u\right)^{2} .
$$

Analogous to the ingoing case, since $H \geq 0$, the hypersurfaces $u=$ const are spacelike hyperbolae which approach $\mathcal{I}^{+}$, except in the limiting Schwarzschild case where they are null. Explicitly, following the calculation of (25),

$$
n^{(-) \alpha} \partial_{\alpha} u=1-\frac{r}{\rho} \text {. }
$$

In order to compactify $\mathcal{I}^{+}$, we replace the hyperboloidal spherical coordinates $\tilde{x}^{(-) \alpha}=\left(u, \rho, \theta, \phi^{(-)}\right)$by the compactified coordinates $\hat{x}^{(-) \alpha}=\left(u, \ell, \theta, \phi^{(-)}\right)$, where $\ell=1 / \rho$, and $\ell=0$ at $\mathcal{I}^{+}$. Again we introduce the conformally rescaled metric $\hat{g}_{a b}=\ell^{2} g_{a b}$,

$$
\hat{g}_{\alpha \beta} d \hat{x}^{(-) \alpha} d \hat{x}^{(-) \beta}=-\ell^{2} d u^{2}+2 d u d \ell+d q^{(-) 2}+2 H\left(\ell n_{\alpha}^{(-)} d \hat{x}^{\alpha(-)}\right)^{2},
$$

where

$$
\ell n_{\alpha}^{(-)} d \hat{x}^{(-) \alpha}=-\ell d u+\left[\ell^{-1}-r\right] d \ell-\frac{a \sin \theta}{r \ell\left(r^{2}+a^{2}\right)}\left(a \cos \theta d \theta-r \sin \theta d \phi^{(-)}\right) .
$$

The asymptotic behaviour of $\hat{g}_{\alpha \beta}$ at $\mathcal{I}^{+}$follows from the asymptotic $\ell$ expansion (31) of the Boyer-Lindquist coordinate $r$ which leads to

$$
\begin{aligned}
\ell n_{\alpha}^{(-)} d \hat{x}^{(-) \alpha} & =\ell\left(-d u+\frac{a^{2}}{2} \sin ^{2} \theta d \ell+a \sin ^{2} \theta d \phi^{(-)}\right)+\ell^{2}\left(-a^{2} \sin \theta \cos \theta d \theta\right) \\
& +\ell^{3}\left[-\frac{a^{4}}{8}\left(5 \cos ^{2} \theta-1\right) \sin ^{2} \theta d \ell-a^{3} \sin ^{2} \theta \cos ^{2} \theta d \phi^{(-)}\right] \\
& +\ell^{4}\left[-\frac{a^{4}}{2}\left(1-3 \cos ^{2} \theta\right) \sin \theta \cos \theta d \theta\right]+O\left(\ell^{5}\right)
\end{aligned}
$$


so that

$$
\begin{aligned}
& \hat{g}_{\alpha \beta} d \hat{x}^{(-) \alpha} d \hat{x}^{(-) \beta}=2 d u d \ell+d q^{(-) 2}-\ell^{2} d u^{2}+2 m \ell^{3}\left(-d u+\frac{a^{2}}{2} \sin ^{2} \theta d \ell+a \sin ^{2} \theta d \phi^{(-)}\right)^{2} \\
& +\ell^{4}\left[-4 m a^{2}\left(-d u+\frac{a^{2}}{2} \sin ^{2} \theta d \ell+a \sin ^{2} \theta d \phi^{(-)}\right) \sin \theta \cos \theta d \theta\right]+O\left(\ell^{5}\right)
\end{aligned}
$$

with determinant $\hat{g}=-\sin ^{2} \theta$ and inverse

$$
\begin{aligned}
& \hat{g}^{\alpha \beta} \partial_{\hat{x}^{(-) \alpha}} \partial_{\hat{x}^{(-) \beta}}=2 \partial_{u} \partial_{\ell}+q^{(-) A B} \partial_{\hat{x}^{(-) A}} \partial_{\hat{x}^{(-) B}}+\ell^{2} \partial_{\ell} \partial_{\ell}-2 m \ell^{3}\left(\frac{a^{2} \sin ^{2} \theta}{2} \partial_{u}-\partial_{\ell}+a \partial_{\phi^{(-)}}\right)^{2} \\
& +4 a^{2} m \ell^{4}\left[\left(\frac{a^{2} \sin ^{2} \theta}{2} \partial_{u}-\partial_{\ell}+a \partial_{\phi^{(-)}}\right) \sin \theta \cos \theta\right] \partial_{\theta}+O\left(\ell^{5}\right) .
\end{aligned}
$$

We have

$$
\left.\hat{g}_{\alpha \beta} d \hat{x}^{(-) \alpha} d \hat{x}^{(-) \beta}\right|_{\mathcal{I}^{+}}=2 d u d \ell+d q^{(-) 2},
$$

i.e. $\mathcal{I}^{+}$is a null hypersurface with standard asymptotically Minkowskian geometry consisting of unit sphere cross-sections. In addition, analogous to the case for $\mathcal{I}^{-}$,

$$
\hat{\nabla}_{\alpha} \hat{\nabla}_{\beta} l_{\mathcal{I}^{+}}=0
$$

so that $\ell$ is a preferred conformal factor in which the shear and divergence of $\mathcal{I}^{+}$vanish.

An important feature is that both $\mathcal{I}^{-}$and $\mathcal{I}^{+}$have universal conformal structure of unit sphere cross-sections with the identical conformal factors $\ell$. Moreover, (15) leads to the expansion

$$
\Psi=-2 m a \ell^{2}-\frac{8}{3} m^{2} a \ell^{3}-2 m a\left(2 m^{2}-a^{2} \cos ^{2} \theta\right) \ell^{4}+O\left(\ell^{5}\right)
$$

so that $\left.\Psi\right|_{\ell=0}=0$. Consequently, (14) and (17) imply that we can set

$$
\phi=\left.\phi^{(+)}\right|_{\ell=0}=\left.\phi^{(-)}\right|_{\ell=0}
$$

and

$$
d q^{2}=q_{A B} d x^{A} d x^{B}=d \theta^{2}+\sin ^{2} \theta d \phi^{2}=\left.d q^{(+) 2}\right|_{\ell=0}=\left.d q^{(-) 2}\right|_{\ell=0} .
$$

Thus we can use a common unit sphere metric $q_{A B}$, with associated covariant derivative $\widetilde{\partial}_{A}$, common spherical coordinates $x^{A}=(\theta, \phi)$ and a common polarisation dyad $q_{A B}=(1 / 2)\left(q_{A} \bar{q}_{B}+\bar{q}_{A} q_{B}\right)$ to describe both the ingoing radiation from $\mathcal{I}^{-}$and the outgoing radiation at $\mathcal{I}^{+}$.

\section{Boosts and radiation memory}

Analogous to (44), the outgoing radiation strain at $\mathcal{I}^{+}$can be described by a spinweight-2 function $\sigma\left(u, x^{A}\right)$, where $u$ is the retarded time and $x^{A}=(\theta, \phi)$ are the angular coordinates on $\mathcal{I}^{+}$determined by the Minkowski background. In these retarded coordinates adapted to the asymptotic Minkowskian structure, the strain is given by the limit at $\mathcal{I}^{+}$, holding $u$ and $x^{A}$ constant,

$$
\sigma\left(u, x^{A}\right)=\lim _{\rho \rightarrow \infty} \frac{1}{2 \rho} q^{A} q^{B} g_{A B}=q^{A} q^{B} \sigma_{A B}\left(u, x^{A}\right) .
$$


The radiation memory $\Delta \sigma\left(x^{C}\right)$ at $\mathcal{I}^{+}$measures the change in the radiation strain between infinite future and past retarded time,

$$
\Delta \sigma\left(x^{A}\right)=\sigma\left(u=\infty, x^{A}\right)-\sigma\left(u=-\infty, x^{A}\right) .
$$

In the associated inertial Cartesian coordinates, the dyad $q^{A}$ has components $q^{i}=\rho Q^{i}$ and

$$
\sigma\left(u, x^{A}\right)=\lim _{\rho \rightarrow \infty} \frac{\rho}{2} Q^{i} Q^{j} g_{i j} .
$$

For the unboosted KSK metric,

$$
\sigma\left(u, x^{A}\right)=\lim _{\rho \rightarrow \infty} \rho H\left(Q^{i} n_{i}^{(-)}\right)^{2}=\lim _{\rho \rightarrow \infty} m\left(Q^{i} n_{i}^{(-)}\right)^{2} .
$$

A straightforward calculation gives

$$
Q^{i} n_{i}^{(-)}=-\frac{\rho a^{2} \sin \theta \cos \theta}{r\left(r^{2}+a^{2}\right)}+\frac{i \rho a \sin \theta}{r^{2}+a^{2}} .
$$

Again using (31), this implies $Q^{i} n_{i}^{(-)}=O(1 / \rho)$ so that the radiation strain at $\mathcal{I}^{+}$of the unboosted KSK metric vanishes.

Consider now a system which is asymptotically described by an unboosted Kerr metric in the retarded past $u=-\infty$ and by a boosted KSK metric in the future $u=\infty$, where the boost $\mathcal{B}$ is a Lorentz symmetry of $\eta_{\alpha \beta}^{(+)}$. The radiation memory due to the boost is then given by

$$
\Delta \sigma\left(x^{A}\right)=\sigma_{\mathcal{B}}\left(u=\infty, x^{A}\right)-\sigma\left(u=-\infty, x^{A}\right),
$$

where $\sigma_{\mathcal{B}}\left(u=\infty, x^{A}\right)$ is the strain of the final boosted state and the intial strain vanishes, $\sigma\left(u=-\infty, x^{A}\right)=0$. The strain $\sigma_{\mathcal{B}}\left(u=\infty, x^{A}\right)$ may be calculated using either the ingoing or outgoing form of the KSK metric. It is technically simpler to use the ingoing form since the boost $\mathcal{B}$ leaves $\eta_{\alpha \beta}^{(+)}$unchanged. The final strain for the boosted version of the KSK metric $g_{\mathcal{B} \alpha \beta}$, computed in the same frame as the initial strain (84), is then given by

$$
\sigma_{\mathcal{B}}\left(u=\infty, x^{A}\right)=\lim _{u \rightarrow \infty} \lim _{\rho \rightarrow \infty} \frac{\rho}{2} Q^{i} Q^{j} g_{\mathcal{B} i j}=\lim _{u \rightarrow \infty} \lim _{\rho \rightarrow \infty} \rho H_{\mathcal{B}}\left(Q^{i} N_{i}\right)^{2},
$$

where $N_{i}=n_{\mathcal{B} i}^{(+)}$. The leading terms in the $1 / \rho$ expansion of $\rho_{\mathcal{B}}, r_{\mathcal{B}}$ and $H_{\mathcal{B}}$, given in (57), (60) and (61), are unchanged when the limit at $\mathcal{I}^{+}$is taken holding $u$ constant. Thus

$$
\sigma_{\mathcal{B}}\left(u=\infty, x^{A}\right)=\lim _{u \rightarrow \infty} \lim _{\rho \rightarrow \infty} \frac{m \rho}{\rho_{\mathcal{B}}}\left(Q^{i} N_{i}\right)^{2} .
$$

The key difference here is that the limit at $\mathcal{I}^{+}$involves the boosted ingoing principal null direction $N_{\alpha}$ whose asymptotic behaviour (63) leads to

$$
Q^{i} N_{i}=Q^{i} v_{i}\left(1+\frac{\Gamma\left(t^{(+)}-V_{i} x^{(+) i}\right)}{\rho_{\mathcal{B}}}\right)+O(1 / \rho) .
$$

Now, instead of holding the advanced time $v$ constant to take the limit at $\mathcal{I}^{-}$, we hold the retarded time $u$ constant to take the limit at $\mathcal{I}^{+}$. Referring to (13)

$$
t^{(+)}=t^{(-)}+2\left(r-r^{*}\right)=u+\rho+2\left(r-r^{*}\right),
$$


where (4) leads to the expansion

$$
r^{*}-r=2 m \ln \left(\frac{\rho}{2 m}\right)-\frac{4 m^{2}}{\rho}+O\left(1 / \rho^{2}\right) .
$$

From (56) $), \rho_{\mathcal{B}}$ has the asymptotic behaviour, holding $u$ constant,

$$
\frac{\rho_{\mathcal{B}}}{\rho}=\Gamma\left(1-\rho^{i} V_{i}\right)+O(1 / \rho),
$$

where $\rho^{i}$ is defined in (58). As a result, since $\lim _{\rho \rightarrow \infty} \ln \rho / \rho=0$, (91) leads to the limit, holding $u$ constant,

$$
\lim _{\rho \rightarrow \infty} \frac{t^{(+)}}{\rho_{\mathcal{B}}}=\lim _{\rho \rightarrow \infty} \frac{\rho}{\rho_{\mathcal{B}}}=\frac{1}{\Gamma\left(1-\rho^{i} V_{i}\right)}
$$

and (90) leads to

$$
\lim _{\rho \rightarrow \infty} Q^{i} N_{i}=2 Q^{i} v_{i} .
$$

Thus

$$
\sigma_{\mathcal{B}}\left(u=\infty, x^{A}\right)=\lim _{u \rightarrow \infty} \lim _{\rho \rightarrow \infty} \frac{4 m \rho}{\rho_{\mathcal{B}}}\left(Q^{i} v_{i}\right)^{2}=\frac{4 m \Gamma}{\left(1-\rho^{i} V_{i}\right)}\left(Q^{i} V_{i}\right)^{2} .
$$

The resulting boost memory due to the ejection of a Kerr black hole of mass $m$ is

$$
\Delta \sigma=\frac{4 m \Gamma}{\left(1-\rho^{i} V_{i}\right)}\left(Q^{i} V_{i}\right)^{2} .
$$

This is identical to the nonlinear result [4] and to the linearised result [2] for the memory due to the relative boost between the initial and final states of a Schwarzschild black hole.

\section{Discussion}

We have shown that a boost $\mathcal{B}$ of the Minkowski background $\eta_{a b}^{(+)}$for the ingoing KSK metric leads to a model for the memory effect for an initially stationary Kerr black hole which, after some accelerating and radiating stage, results in a final boosted state. The result is consistent with the absence of ingoing radiation and does not depend upon any linearised approximation. The full memory effect combines the boost memory, which we now denote by $\Delta \sigma_{\mathcal{B}}$, and the null memory $\Delta \sigma_{\mathcal{N}}$ resulting from radiative energy loss to $\mathcal{I}^{+}$. The net memory effect is then

$$
\Delta \sigma=\Delta \sigma_{\mathcal{B}}+\Delta \sigma_{\mathcal{N}}
$$

Although we have concentrated on the boost memory for the simple process consisting of the ejection of a boosted Kerr black hole, the result can be generalised. First, the asymptotic Lorentz symmetry at null infinity implies that the radiation memory for the transition of a black hole from a rest state to a boosted state with mass $m$ and velocity $V^{i}$ is the same as the memory for a black hole of mass $m$ with initial velocity $-V^{i}$ and zero final velocity. In addition, even in the nonlinear theory it is expected that the superposition principle holds for particles at infinite separation since 
the constraints vanish in that limit. This allows the memory effect to be generalised to a system of particles.

As a simple example, we consider the collision of two distant Kerr black holes of mass $m$ with initial velocities $V^{i}$ and $-V^{i}$ in the $z$-direction which come to rest in a final state with mass $M$. The collision is constrained by the Bondi mass loss fomula, which requires

$$
2 m \Gamma-M=\int_{-\infty}^{\infty} d u \oint\left\|\partial_{u} \sigma\right\|^{2} \sin \theta d \theta d \phi
$$

The null memory is determined nonlocally from the integrated radiation flux. In the case of gravitational radiation, Christodoulou's result [10] can be expressed in terms of the radiation strain according to [19, 4]

$$
\bar{q}^{A} \bar{q}^{B} \varlimsup_{A} ð_{B} \Delta \sigma_{\mathcal{N}}=\int_{-\infty}^{\infty}\left\|\partial_{u} \sigma\right\|^{2} d u-\Delta \mathcal{P}
$$

where $\Delta \mathcal{P}$ cancels the $l=0$ and $l=1$ spherical harmonics in the flux integral. The $l=0$ harmonic of the integrand determines the mass loss via (99) and the $l=1$ harmonics determine the momentum loss. These harmonics do not enter the memory effect which is a spin-weight-2 quantity with $l \geq 2$.

According to (97), the boost memory for this process is

$$
\Delta \sigma_{\mathcal{B}}=4 m \Gamma\left(Q^{i} V_{i}\right)^{2}\left(\frac{1}{1+\rho^{i} V_{i}}+\frac{1}{1-\rho^{i} V_{i}}\right)=\frac{8 m \Gamma V^{2} \sin ^{2} \theta}{1-V^{2} \cos ^{2} \theta} .
$$

As a consequence of (99), $2 m \Gamma-M>0$ so that the boost memory (101) for the collision has a lower bound determined by the mass of the final black hole,

$$
\Delta \sigma_{\mathcal{B}}>\frac{4 M V^{2} \sin ^{2} \theta}{1-V^{2} \cos ^{2} \theta}
$$

This lower bound is largest when the merger of the two black holes takes place slowly so that $M \approx 2 m \Gamma$ and there is negligible radiative energy loss and negligible null memory.

The memory effect is also constrained by Hawking's area increase law for the event horizon in the merger of two black holes [28]. For the above collision of initially distant Schwarzschild black holes to form a Kerr black hole, this leads to

$$
4 m^{2}<M\left(M+\sqrt{M^{2}-A^{2}}\right)=M^{2}(1+\cos \chi), \quad 0 \leq \chi \leq \pi / 2,
$$

where $A=M \sin \chi$ is the specific angular momentum of the final Kerr black hole as determined by the initial impact parameter. Thus (103) implies

$$
2 m<M \sqrt{1+\cos \chi} .
$$

As a result, the boost memory (101) has both upper and lower bounds in terms of the final black hole mass,

$$
\frac{4 M \Gamma V^{2} \sin ^{2} \theta \sqrt{1+\cos \chi}}{1-V^{2} \cos ^{2} \theta}>\Delta \sigma_{\mathcal{B}}>\frac{4 M V^{2} \sin ^{2} \theta}{1-V^{2} \cos ^{2} \theta} .
$$

There is no universal way to separate the net memory effect into boost and null mechanisms without knowledge of the strain in the intermediate radiative period. 
However, there are two extremes in which the net memory takes either the pure boost or pure null form. The first is the adiabatic limit, discussed in [4], in which the intermediate processes take place slowly and $\Delta \sigma_{\mathcal{N}}$ is negligible. This limit was first considered in [31] and is now referred to in the literature as the infrared or "soft graviton" limit [32, 33]. In the other extreme, no boosted massive particles are captured or ejected from the intrinsic rest frame of the system, so that $\Delta \sigma=\Delta \sigma_{\mathcal{N}}$. An example would be a binary in an initial Newtonian orbit which subsequently inspirals to form a stationary black hole with respect to the initial rest frame.

In [4, we analysed how radiation memory affects angular momentum conservation. In a non-radiative regime where $\partial_{u} \sigma=0$, the supertranslation freedom (46) can be used to pick out preferred cross-sections of $\mathcal{I}^{+}$by setting the electric component of $\sigma$ to 0 . These preferred cross-section reduce the supertranslation freedom to the translation freedom so that a preferred Poincaré subgroup can be picked out from the BMS group. The same holds in the limits $u \rightarrow \pm \infty$, in which the requirement of a finite radiative energy loss implies $\partial_{u} \sigma \rightarrow 0$. Although the electric part of the strain can be gauged away at either $u=+\infty$ or $u=-\infty$, the memory effect $\Delta \sigma$ is gauge invariant and (46) determines a supertranslation shift

$$
q^{A} q^{B} \check{\partial}_{A} ð_{B} \alpha\left(x^{C}\right)=\Delta \sigma\left(x^{C}\right)
$$

between the preferred Poincaré groups at $u= \pm \infty$. The rotation subgroups picked out by the initial and final preferred Poincaré groups differ by this supertranslation. As a result, the corresponding components of angular momentum intrinsic to the initial and final states differ by supermomenta.

Only the electric part of the strain is affected by supertranslations because $\alpha$ is real and $\sigma$ is intrinsically complex. The decomposition of the strain into electric and magnetic parts is analogous to the E-mode/B-mode decomposition of electromagnetic waves. The magnetic part of the null memory effect must vanish except for matter fields whose stress-energy tensor satisfies properties which are not expected for astrophysical systems [3]. The exceptional cases are matter fields whose magnetic part of their stress is anisotropic and has nonvanishing time derivative in the limit of infinite future or past retarded time in the neighbourhood of $\mathcal{I}^{+}$. Recently, consistent with general physical principles, a shell of matter with anisoptropic magnetic stress which expands to timelike infinity in a radial flow has been constructed which produces ordinary memory of the magnetic type [34].

The supertranslation shift between the initial and final preferred Poincaré groups complicates the interpretation of angular momentum flux conservation laws. This could lead to a distinctly general relativistic mechanism for angular momentum loss. Although the intermediate radiative epoch must be treated by numerical methods, the Kerr-Schild model developed here provides a framework for such investigations. 


\section{Acknowledgments}

We are grateful to the AEI in Golm for hospitality during this project. We thank I. Rácz for comments on the manuscript and R. M. Wald for informing us of recent results. TM appreciates support from the members of the Núcleo de Astronomía and Faculty of Engineering of University Diego Portales, Santiago. JW was supported by NSF grants PHY-1505965 and PHY-1806514 to the University of Pittsburgh.

\section{References}

[1] Zeldovich Y and Polnarev A G 1974 Radiation of gravitational waves by a cluster of superdense stars Sov. Astron. 1817

[2] Braginsky V B and Thorne K S 1987 Gravitational wave bursts with memory and experimental prospects Nature (London) $\mathbf{3 2 7} 123$

[3] Mädler T and Winicour J 2016 The sky pattern of the linearized gravitational memory effect Class. Quantum Grav. 33175006

[4] Mädler T and Winicour J 2017 Radiation memory, boosted Schwarzschild spacetimes and supertranslations Class. Quantum Grav. 34115009

[5] Mädler T and Winicour J 2018 Boosted Schwarzschild metrics from a Kerr-Schild perspective Class. Quantum Grav. 35035009

[6] Kerr R P and Schild A 1965 Some algebraically degenerate solutions of Einstein's gravitational field equations Proc. Symp. Appl. Math. 17199

[7] Kerr R P and Schild A 1966 A new class of vacuum solutions of the Einstein field equations Atti degli Convegno Sulla Relativita Generale p. 222 (Firenze) [online as Kerr R P and Schild A Republication of: A new class of vacuum solutions of the Einstein field equations (2009) Gen. Rel. Grav. 412485 ]

[8] Boyer R H and Lindquist R W 1967 Maximal Analytic Extension of the Kerr Metric J. Math. Phys. 8265

[9] Sachs R 1962 Asymptotic symmetries in gravitational theory Phys. Rev. 1282851

[10] Christodoulou D 1991 Nonlinear nature of gravitation and gravitational-wave experiments Phys. Rev. Letters 671486

[11] Friedrich H 1983 Cauchy problems for the conformal vacuum field equations in general relativity Comm. Math. Phys. 91445

[12] Zenginoğlu A 2008 Hyperboloidal foliations and scri-fixing Class. Quantum Gravity 25145002

[13] Zenginoğlu A 2011 A geometric framework for black hole perturbations Phys. Rev. D 83127502

[14] Bieri L and Garfinkle D 2014 Perturbative and gauge invariant treatment of gravitational wave memory Phys. Rev. D 89084039

[15] Bieri L, Chen P and Yau S T 2012 The electromagnetic Christodoulou memory effect and its application to neutron star binary mergers Class. Quantum Gravity 2921

[16] Bieri L and Garfinkle D 2015 Neutrino radiation showing a Christodoulou memory effect in general relativity Ann. Henri Poincaré 16801

[17] Tolish A and Wald R M 2014 Retarded field of null particles and the memory effect Phys. Rev. D 89064008

[18] Bieri L, Chen P and Yau S T 2011 Null asymptotics of solutions of the Einstein-Maxwell equations in general relativity and gravitational radiation Adv. Theor. Math. Phys. 154

[19] Frauendiener J 1992 Note on the memory effect Class. Quantum Gravity 91639

[20] Pasterski S Strominger A and Zhiboedov A 2015 New Gravitational Memories eprint arXiv:1502.06120

[21] Flanagan E and Nichols D A 2015 Observer dependence of angular momentum in general relativity and its relationship to the gravitational-wave memory effect; Phys. Rev. D 92, 084057 
[22] Nichols D A 2018 Center-of-mass angular momentum and memory effect in asymptotically flat spacetimes Phys. Rev. D 98064032

[23] Kramer D, Stephani H, MacCallum M and Herlt E 1980 Exact solutions of Einstein's field equations, ed. Schmutzer E (Cambridge University Press, Cambridge)

[24] Matzner R A, Huq M F and Shoemaker D 1998 Initial data and coordinates for multiple black hole systems Phys. Rev. D 59024015

[25] Bonning E, Marronetti P, Neilson D, and Matzner R A 2003 Physics and initial data for multiple black hole spacetimes Phys. Rev. D 68044019

[26] Mädler T and Winicour J 2016 Bondi-Sachs formalism Scholarpedia. 11 (12) 33528

[27] Geroch R and Winicour J 1981 Linkages in general relativity J. Math. Phys. 22803

[28] Hawking S W 1971 Gravitational radiation from colliding black holes Phvs. Rev. Letters 261344

[29] Tamburino L A and Winicour J 1966 Gravitational fields in finite and conformal Bondi frames Phys. Rev. 1501039

[30] Winicour J 2014 Global aspects of radiation memory Class. Quantum Grav. 31205003

[31] Smarr L 1977 Gravitational radiation from distant encounters and from head-on collisions of black holes - The zero-frequency limit Phys. Rev. D 152069

[32] Pasterski S 2017 Asymptotic symmetries and electromagnetic memory J. High Energ. Phys. 154

[33] Strominger A and Zhiboedov A 2016 Gravitational memory, BMS supertranslations and soft theorems J. High Energy Phys. 86

[34] Satishchandran G and Wald R M 2019 The asymptotic behavior of massless fields and the memory effect http://arxiv.org/abs/1901.05942 